\title{
LINEAR INDEPENDENCE OF ITERATES OF ENTIRE FUNCTIONS
}

\author{
LUIS BERNAL GONZÁLEZ
}

(Communicated by Paul S. Muhly)

\begin{abstract}
We prove the following result: The set $\left\{h_{n}: n=0,1, \ldots\right\}$ is a linearly independent sequence of entire functions, where $h_{0}=1, h_{1}=g_{1}$, $h_{2}=g_{1} \circ g_{2}, h_{3}=g_{1} \circ g_{2} \circ g_{3}, \ldots, g_{1}$ is a nonconstant entire function and $g_{n} \quad(n \geq 2)$ are entire functions which are not polynomials of degree $\leq 1$. Our theorem generalizes a previous one about linear independence of iterates.
\end{abstract}

In [1] it is proved that except for trivial cases a sequence of iterates of entire functions is always linearly independent. As a related result, it is also shown in [1] that the Feigenbaum functional equation:

$$
\begin{array}{cc}
f(f(\lambda x))+\lambda f(x)=0 \quad & (-1 \leq x \leq 1) ; \\
0<\lambda=-f(1)<1 ; & f(0)=1,
\end{array}
$$

does not have an entire solution.

In this paper we shall generalize the interesting theorems proved in [1]. For the sake of completeness, we state them. In the following we denote by $\mathscr{F}$ the space of all entire functions, and by $\mathbf{C}$ the complex plane.

Theorem A. Let $f$ be a nonidentically zero entire function that satisfies the functional equation:

$$
f(f(\lambda z))+\lambda f(z)=0
$$

for all $z \in \mathbf{C}$, where $\lambda$ is a fixed nonzero complex number, then either $f(z)=-z$ and $\lambda$ is arbitrary or $f(z)$ is a constant with $\lambda=-1$.

From Theorem A, the nonexistence of entire solutions of (1) is derived.

Theorem B. Let $f$ be an entire function that is not a polynomial of degree $\leq 1$. Let $R_{f}: \mathscr{F} \rightarrow \mathscr{F}$ be defined by $R_{f}(g)=g \circ f$ for all $g \in \mathscr{F}$. Then $R_{f}$ has no eigenvalue distinct from 0,1 and any eigenfunction with eigenvalue 1 is a constant.

Theorem C. Let $g$ be a nonconstant entire function and let $f$ be an entire function which is not a polynomial of degree $\leq 1$. Denote the successive iterates

Received by the editors February 12, 1990.

1980 Mathematics Subject Classification (1985 Revision). Primary 30D05; Secondary 30D15.

Key words and phrases. Entire functions, linear independence, iterates, maximum modulus, asymptotic inequalities, composite functions. 
of $f$ in the following way: $f_{1}=f, f_{2}=f \circ f_{1}, \ldots, f_{n+1}=f \circ f_{n}(n=1,2, \ldots)$, and $f_{0}$ will stand for the identity mapping. Then $\left\{g \circ f_{n}: n \geq 0\right\}$ is a linearly independent sequence of entire functions.

The proofs of these results are based upon a classical result of Pólya (see [5; 2 , pp. 50-51]) concerning the growth of Nevanlinna's characteristics of composite functions. We prove a more general theorem without using these characteristics.

First, we need several results about the maximum modulus of an entire function. Throughout this paper we use the following notation. If $f, g, h, f_{i}, g_{i}$, $h_{i}, \ldots$ are entire functions, then $F, G, H, F_{i}, G_{i}, H_{i}, \ldots$ respectively are their maximum modulus functions, i.e., $F(r)=\max \{|f(z)|:|z|=r\} \quad(r>0)$ and so on. If $f=\sum_{i=1}^{n} \lambda_{i} f_{i}\left(\lambda_{i} \in \mathbf{C} ; i=1,2, \ldots, n\right)$ and $h=g \circ f$, then, trivially, $F(r) \leq \sum_{i=1}^{n}\left|\lambda_{i}\right| F_{i}(r)$ and $H(r) \leq G(F(r))$ for all $r>0$. For functions defined on large enough $r>0$, we say that $\varphi(r)<\psi(r)$ asymptotically (asymp.) when there is $r_{0}>0$ with $\varphi(r)<\psi(r)$ for every $r>r_{0}$.

The proof of the following theorem can be found, for instance, in [3, pp. $80-81 ; 4$, pp. 225-227].

Theorem D. Assume that $f, g \in \mathscr{F}, h=g \circ f$, and $f(0)=0$. Then, there exists $c \in(0,1)$ independent from $f$ and $g$ such that

$$
G(c F(r / 2)) \leq H(r) \text { for all } r>0 \text {. }
$$

Next, we state two previous lemmas.

Lemma 1. Suppose that $f \in \mathscr{F}, f$ is not constant, $\alpha>1$ and $0<\beta<\alpha$. Then $\beta F(r)<F(\alpha r)$ asymp.

Proof. We obtain $|g(z)| \leq|z| G(R) / R$ if $|z| \leq R$ by applying Schwarz's lemma to $g(z)=f(z)-f(0)$. If $R=\alpha r$, then $F(r)-|f(0)| \leq G(r) \leq(r / R)(F(R)+$ $|f(0)|)=(F(\alpha r)+|f(0)|) / \alpha$. Choose $\varepsilon=(\alpha-\beta) /(2 \alpha+2 \beta)$. Then there is $r_{0}>0$ with $|f(0)|<\varepsilon F\left(r_{0}\right)$. Thus $F(\alpha r) \geq((1-\varepsilon) \alpha /(1+\varepsilon)) F(r)>\beta F(r)$ for all $r>r_{0}$.

Lemma 2. Assume that $p$ is a nonnegative integer, $p \geq 2, g_{i} \in \mathscr{F} \quad(i=$ $1,2, \ldots, p), g_{i}$ is a nonconstant function $(i=2,3, \ldots, p)$ and $h=g_{1} \circ g_{2} \circ$ $\cdots \circ g_{p}$. Then, there exists $d \in(0,1)$ such that

$$
H(r) \geq\left(G_{1} \circ G_{2} \circ \cdots \circ G_{p}\right)(d r) \text { asymp. }
$$

Proof. Let us prove the lemma for $p=2$ and put $g(z)=g_{1}\left(z+g_{2}(0)\right)$ and $f(z)=g_{2}(z)-g_{2}(0)$. Evidently, $h=g \circ f, f(0)=0, F(r) \geq G_{2}(r)-\left|g_{2}(0)\right|$ $(r>0), G(s) \geq G_{1}\left(s-\left|g_{2}(0)\right|\right) \quad\left(s>\left|g_{2}(0)\right|\right)$ and $G_{2}(r)>(2+(2 / c))\left|g_{2}(0)\right|$ asymp., where $c$ is the constant in Theorem $\mathrm{D}$. Then by this theorem we can conclude that

$$
G(c F(r / 2)) \geq G_{1}\left(c F(r / 2)-\left|g_{2}(0)\right|\right) \geq G_{1}\left(c G_{2}(r / 2)-(c+1)\left|g_{2}(0)\right|\right) .
$$

Hence

$$
H(r) \geq G_{1}\left((c / 2) G_{2}(r / 2)\right) \quad \text { asymp }
$$


Let $d=c / 6$. By applying Lemma 1 to $\alpha=3 / c, \beta=2 / c$ we obtain $G_{2}(r / 2)=G_{2}(\alpha d r) \geq \beta G_{2}(d r)$ asymp. This inequality and (2) complete the case $p=2$.

Let us go on by induction. Assume that there is $d_{1} \in(0,1)$ such that

$$
Q(r) \geq\left(G_{1} \circ G_{2} \circ \cdots \circ G_{p-1}\right)\left(d_{1} r\right) \text { asymp. }
$$

where $q(z)=g_{1} \circ g_{2} \circ \cdots \circ g_{p-1}$. We have $h=q \circ g_{p}$, so we can apply the case $p=2$. There exists $d_{2} \in(0,1)$ such that

$$
H(r) \geq Q\left(G_{p}\left(d_{2} r\right)\right) \quad \text { asymp. }
$$

Again apply Lemma 1 with $\alpha=2 / d_{1}, \beta=1 / d_{1}$ and set $d=d_{1} d_{2} / 2$. Then

$$
d_{1} G_{p}\left(d_{2} r\right)=(1 / \beta) G_{p}(\alpha d r) \geq G_{p}(d r) \text { asymp. }
$$

Equations (3)-(5) complete the proof.

Theorem. Suppose that $g_{1}$ is a nonconstant entire function and $g_{n}(n \geq 2)$ are entire functions which are not polynomials of degree $\leq 1$. Define $h_{0}=1$, $h_{1}=g_{1}, h_{n}=h_{n-1} \circ g_{n}(n \geq 2)$. Then $\left\{h_{n}: n=0,1,2, \ldots\right\}$ is a linearly independent sequence of entire functions.

Proof. By contradiction, let $p$ be an integer $\geq 2$ and let $\sum_{i=0}^{p} \lambda_{i} h_{i}(z)=0$ for all $z \in \mathbf{C}$, with $\lambda_{i} \in \mathbf{C}(i=0,1, \ldots, p)$ and $\lambda_{p} \neq 0$ (cases $p=0$ and $p=1$ are trivial). Then $h_{p}=\sum_{i=0}^{p-1} \mu_{i} h_{i}$, where $\mu_{i}=-\lambda_{i} / \lambda_{p}(i=0,1, \ldots, p-1)$. We have $H_{p}(r)<\mu \cdot \sum_{i=0}^{p-1} H_{i}(r) \quad(r>0)$, with $\mu$ a fixed positive real number greater than $\max \left\{\left|\mu_{i}\right|: i=0,1, \ldots, p-1\right\}$. By applying Lemma 2 , we have $\left(G_{1} \circ G_{2} \circ \cdots \circ G_{p}\right)(d r)<\mu+\mu \sum_{i=1}^{p-1}\left(G_{1} \circ G_{2} \circ \cdots \circ G_{i}\right)(r)$ asymp. Define $\varphi$ by

$$
\varphi(r)=\left(G_{1} \circ G_{2} \circ \cdots \circ G_{p-1}\right)(r) \quad(r>0) .
$$

Evidently, $1<\varphi(r)$ asymp. and $\left(G_{1} \circ G_{2} \circ \cdots \circ G_{i}\right)(r) \leq \varphi(r)$ asymp. $(i=$ $1,2, \ldots, p-1)$, because $G_{j}(r)>r$ asymp. for all $j \geq 2$ and each $G_{j}(j \geq 1)$ is strictly increasing, by hypothesis. Hence,

$$
\varphi\left(G_{p}(d r)\right)<p \mu \varphi(r) \quad \text { asymp. }
$$

Since $g_{p}$ is not a polynomial of degree $\leq 1$, the inequality

$$
G_{p}(r)>(p / d)(1+\mu) r \text { asymp. }
$$

holds. Then we apply Lemma $1 p-1$ times to obtain $\varphi\left(G_{p}(d r)\right)>$ $\varphi((p+p \mu) r)>\left(G_{1} \circ G_{2} \circ \cdots \circ G_{p-2}\right)\left((p-1+p \mu) G_{p-1}(r)\right)>\left(G_{1} \circ \cdots \circ G_{p-3}\right)$ $\left((p-2+p \mu)\left(G_{p-2} \circ G_{p-1}\right)(r)\right)>\cdots>(1+p \mu)\left(G_{1} \circ \cdots \circ G_{p-1}\right)(r)>p \mu \varphi(r)$ asymp., that is a contradiction with (6).

Finally, we derive Theorems A, B, and C as a corollary.

Proof of Theorem A. If $f$ is a polynomial of degree $\leq 1$, then the statement is obvious. Now, let $f$ be an entire function that is not a polynomial of degree $\leq 1$. Then $f$ does not satisfy $f(f(\lambda z))+\lambda f(z)=0(z \in \mathbb{C})$ for any nonzero 
$\lambda \in \mathbb{C}$. To see this, we only have to apply our theorem to $g_{1}=f, g_{n}=$ $f(\lambda z)(n \geq 2)$.

Proof of Theorem B. Let $g$ be an eigenfunction for $R_{f}$. Applying our theorem to $g_{1}=g, g_{n}=f \quad(n \geq 2)$, we deduce that $g$ is a constant $(g(z)=\mu \neq 0)$. Consequently, there is $\lambda \in \mathbf{C}$ such that $\mu-\lambda \mu=0$. Thus, in fact, $\lambda=1$ is the unique eigenvalue of $R_{f}$ and the eigenfunctions are precisely the set of all nonzero constant entire functions.

Proof of Theorem C. Again apply our theorem to $g_{1}=g, g_{n}=f(n \geq 2)$.

\section{REFERENCES}

1. J.P.R. Christensen and P. Fischer, Linear independence of iterates and entire solutions of functional equations, Proc. Amer. Math. Soc. 103 (1988), 1120-1124.

2. W. K. Hayman, Meromorphic functions, Oxford Mathematical Monograph, Clarendon Press, 1964.

3. A. S. B. Holland, Introduction to the theory of entire functions, Academic Press, New York and London, 1973.

4. J. E. Littlewood, Lectures on the theory of functions, Oxford Univ. Press, London and New York, 1944.

5. G. Pólya, On an integral function of an integral function, J. London Math. Soc. 1 (1926), $12-15$.

Departamento de Análisis Matemático, Facultad de Matemáticas, Avenida Reina Mercedes, 41080-SeVilla, Spain 